\title{
Software Details in Occlusal Splint Creation through 3Shape Design Studio
}

\author{
Dobromira Shopova $^{1 *(\mathbb{D})}$, Miroslava Yordanova² ${ }^{2}$, Svetlana Yordanova $^{2}$ (D) \\ ${ }^{1}$ Department of Prosthetic Dentistry, Faculty of Dental Medicine, Medical University, Plovdiv, Bulgaria; ${ }^{2}$ Associate Professor in \\ the Department of Orthodontics, Faculty of Dental Medicine, Medical University, Plovdiv, Bulgaria
}

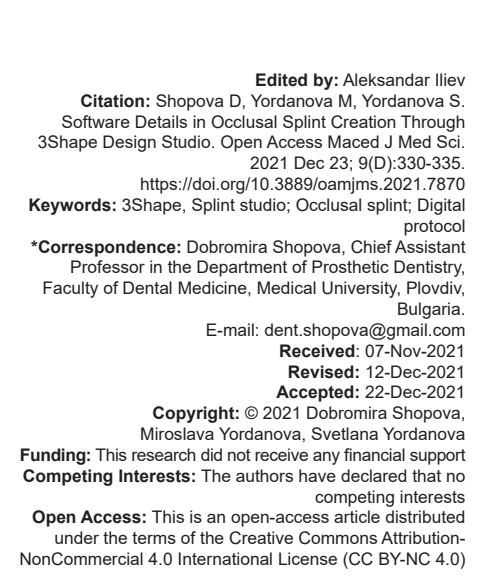

\begin{abstract}
BACKGROUND: Occlusal splints are usually the first choice in the treatment of bruxism. Splints can also be the only treatment or can be ended with adhesive restoration, orthodontic, or prosthetic treatment.

METHODS: This article demonstrates the steps of a digital workflow through the 3Shape Design system - splin studio. The initial units are virtual models, scanned by Trios Color Scanner (3Shape).

RESULTS: The digital protocol includes eight steps that allow precise and detailed creation of the searched object. Each step is described from the perspective of a clinician, prosthetist and orthodontist, not a dental technician. Possible complications and ways to management with them are described.

CONCLUSION: Digital protocol of occlusal splint creation gives many opportunities according to design, thickness and occlusion.
\end{abstract}

\section{Introduction}

Occlusal splints are usually the first choice in the treatment of bruxism. By the term "occlusal splint" or "occlusal device" is meant a removable artificial occlusal surface of plastic or metal that the patient wears temporarily to alter the occlusal contacts and the functional pattern of the mandible. Occlusal splints attempt to create equal contacts of the posterior teeth and to secure the centric or therapeutic mandibular position through maximal intercuspation against the splint [1].

Occlusal splints can be effectively used for the following purposes, according to Clark, Lotzmann and Okeson [2], [3], [4]:

- $\quad$ Improving neuromuscular coordination
- $\quad$ Treatment of myogenic pain
- $\quad$ Treatment of pain arising from the joints
- $\quad$ Increasing the vertical dimension
- $\quad$ Securing a definite mandibular position
- $\quad$ Testing the planned occlusal scheme in centric
and eccentric positions
Splinting of loose teeth

- $\quad$ Distributing the load in bruxism

- Clarifying occlusal etiological factors while making a differential diagnosis.

According to Lotzmann, the multitude of occlusal devices described in the literature can be classified according to their indications as follows [5]:

1. Occlusal devices that are intended primarily to normalize muscle tone and improve neuromuscular coordination (relaxation splints, vertical dimension splints).

2. Occlusal devices that serve primarily to reposition the mandible and decompress joint structures (repositioning splint, decompression splint, vertical dimension splint).

3. Occlusal devices to stabilize the jaw relations that were established during the initial course of occlusal therapy, and to determine the most favorable centric and eccentric occlusal scheme for the final restorations (decompression splint, stabilization splint).

Digital software has further developed its capabilities and the creation of occlusal splints now is possible. Large manufacturers of dental equipment are constantly updating their software [6], [7]. To cover all parameters of splints, it is necessary to perform 
additional extraoral measurements of the position of the lower jaw and its movement during opening and closing (deviation), condyle inclination, Bennett angle, CBCT showing the condition of the anatomical structures of the TMJ and others. All these additional measurements must be in a compatible file format and allow the incorporation of the files into each other [8], [9]. The created digital objects can be realized through the two main methods of laboratory digital equipment - additive manufacturing and CAD/CAM milling. Additive manufacturing or 3D printing creates the object layer by layer, CAD/CAM technology cuts the object from a disks, cubes and others [10], [11].

The purpose of this article is to demonstrate the digital workflow in splint creation through 3Shape Design system - splint studio.

\section{Methods}

Before starting with the design it is necessary to create virtual models. There are two main ways - intraoral scanning of the patient and laboratory scanning of plaster models. The proposed design protocol does not differ, but for more accurate results, intraoral scanning is preferred. Our patients are scanned through a Trios Color scanner (3Shape). After the personal information of the patient, the established sequence and the "splint" option for creating the order was observed. Here can be included more information about the material, color and delivery date. The upper jaw was marked as supporting the splint. The scan started from the lower jaw (antagonists), followed by the upper jaw, left and right bite. The created virtual models were sent as an STL file by e-mail to the laboratory.

3Shape Dental system design - splint studio was used for file processing and creation of the splint design.

\section{Results}

The digital protocol includes a series of eight steps. After confirming the previous, it moves to the next. The return to the previous step, even to the first, is unobstructed, the changes are saved automatically and the work done is not lost. The steps are the following sequence:

\section{Prepare - shaping the model and placing an occlusal plane}

The shaping of the model can be done by cutting out excess soft tissue, which often causes preliminary contacts and distance between the dental arches.

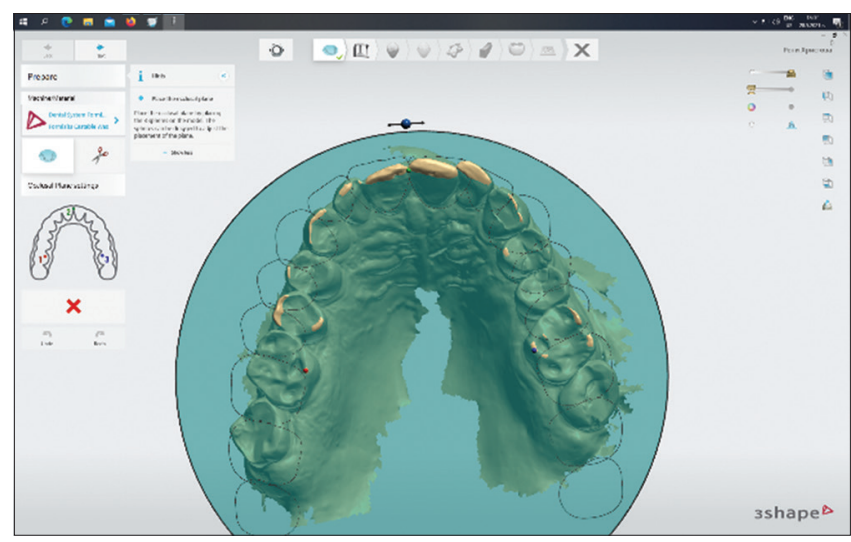

Figure 1: 3-point orientation of the plane

The occlusal plane is oriented by three points - two on the mediopalatal cusps of the first upper molar and one on the contact point of the incisors. Once positioned relative to the dental arch, its horizontal position must also be determined. With the instruments located on the side of the marked circle, the plane can approach or move away from the occlusal surfaces to a satisfactory result. This occlusal plane is important at a later stage in the creation of the occlusal surfaces of the splint, Figures 1 and 2:

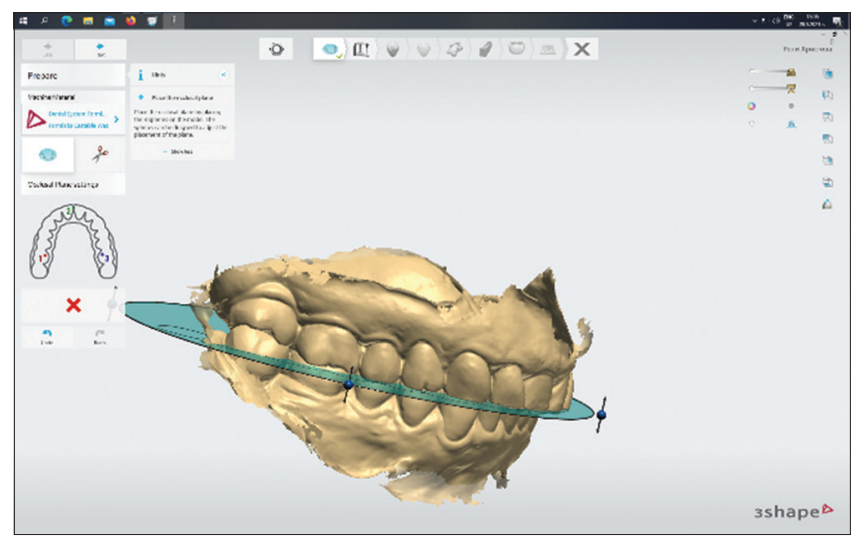

Figure 2: Horizontal position of the plane

\section{Bite configuration}

This step of setting the intermaxillary relationship is basic. By changing the position between the upper and lower jaws, a precise analysis of the occlusion, the lost height, the position of the teeth relative to the horizontal plane can be made. According to this the choice can be directed to a specific type of splint (stabilizing, repositioning, anterior or posterior bite plane, etc.). If there is a distance between the dentitions in its initial position, the error must be sought. It is possible that this distance is from a bad scan - patients do not squeeze the same force when scanning the bite and relax the lower jaw after some seconds. The error may be due to an extensive scan of the surrounding soft tissues to show 
prior contact in the bite. If so, you can go back to the first step, crop the pattern, and reload the images.

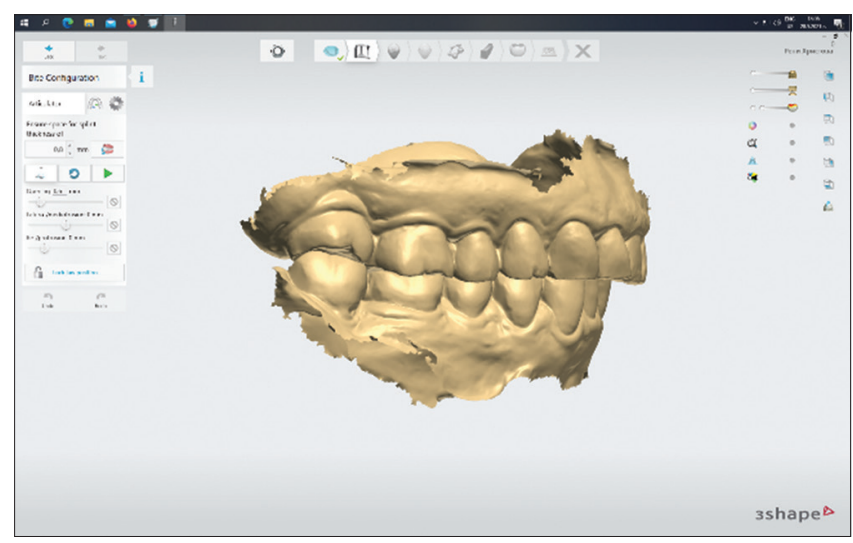

Figure 3: Initial position

Possible directions of movement are: opening, latero-/mediotrusion, re-/protrusion. It is also required to set the thickness of the splint, which the software places at the distal point and opens automatically as needed. If the established opening is more than $5 \mathrm{~mm}$, the thickness of the splint can be reduced (even to the minimum according to the material and methodology which will be used). In situations of higher cusps, pre-leveling of the teeth is required. After establishing the desired position, it should be locked and proceed to the next step, Figures 3 and 4:

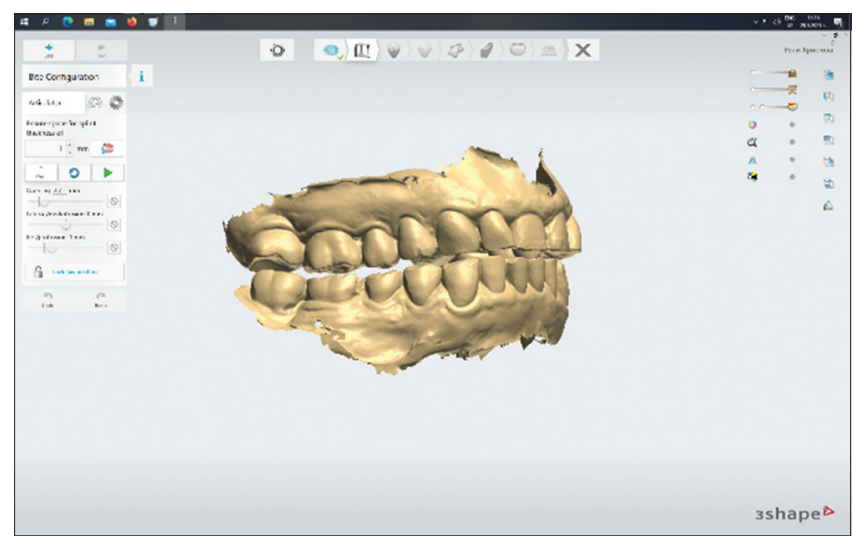

Figure 4: Locked position

For more precise work, the facebow measurements and the virtual articulator can be used. It has set averages, but these parameters can be changed individually. In the absence of such measurements, this step is omitted, Figures 5 and 6:

\section{Blocking out and insertion direction}

The insertion direction is obtained automatically by the system and is averaged between the axes of all teeth. Aims to obtain fewer retention zones. When designing, these areas are visible and it is good for the splint to reach but not pass into them. The software also allows the manual adjustment of the direction. The "retention" option is set individually and shows above

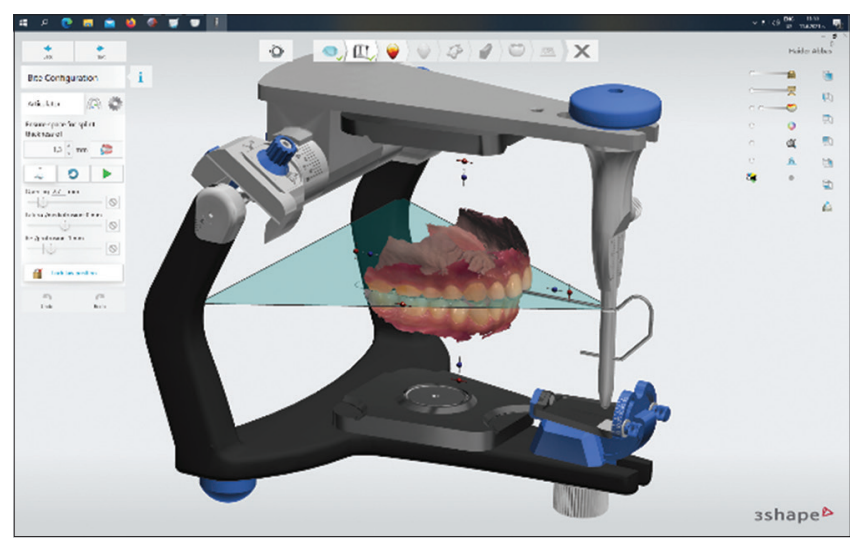

Figure 5: Virtual articulator

what value (eg $0.2 \mathrm{~mm}$ ) the software accepts a section as retention, Figures 7 and 8:

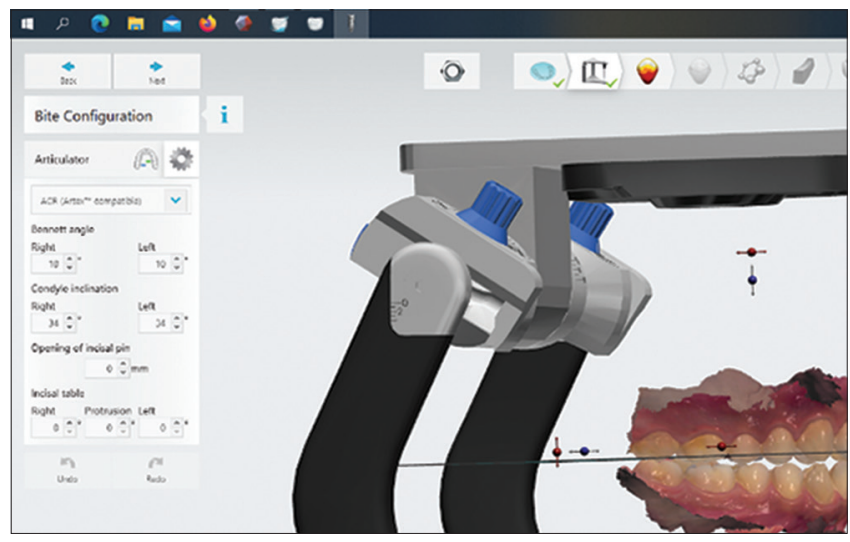

Figure 6: Individual parameters of the atriculator

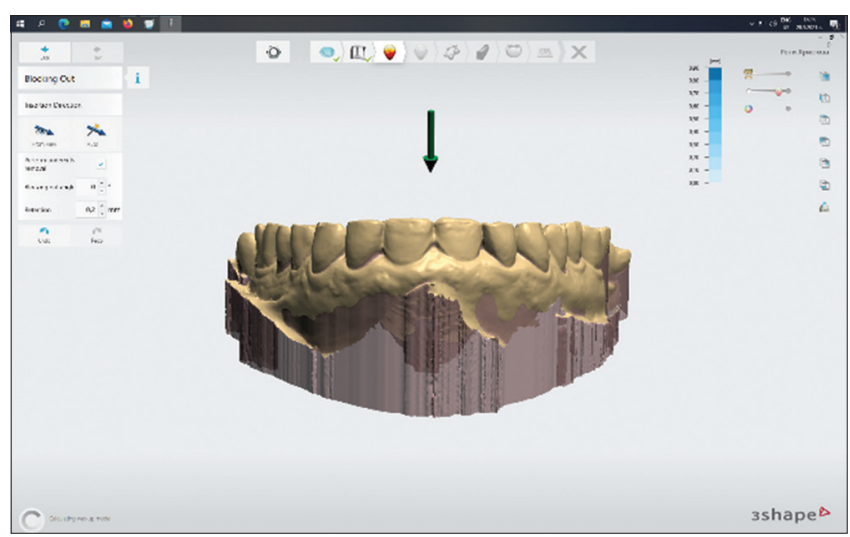

Figure 7: Frontal view of insertion direction

\section{Wax trimming}

This step serves to analyze the models. Slight adjustments to the model can be made by adding, removing, or smoothing. Most often, these are irregularities from scanned saliva or plaque on the tooth surface, which should be leveled for better adaptation. In highly retention areas, "wax" can be added similar to the classic wax modeling work. If no defects are found without making corrections, proceed to the next step, Figure 9: 


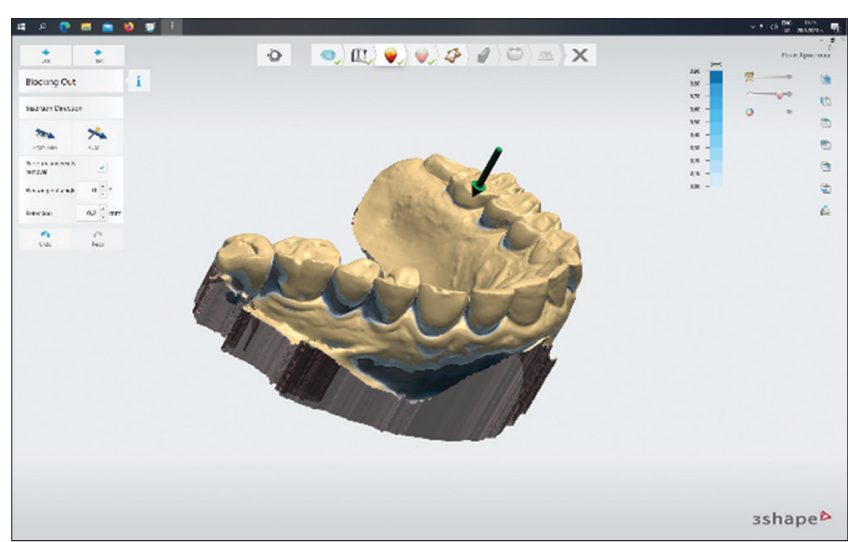

Figure 8: Side view of insertion direction

\section{Outline the boundaries of the splint}

If there is no specific design to adhere to (eg $1 \mathrm{~mm}$ from the incisal edges frontally and $1 \mathrm{~mm}$ apically at the distal teeth), the moderator can make the boundaries at his own discretion and quite individually depending on the insertion direction. The line is drawn by successive points or by moving the mouse.

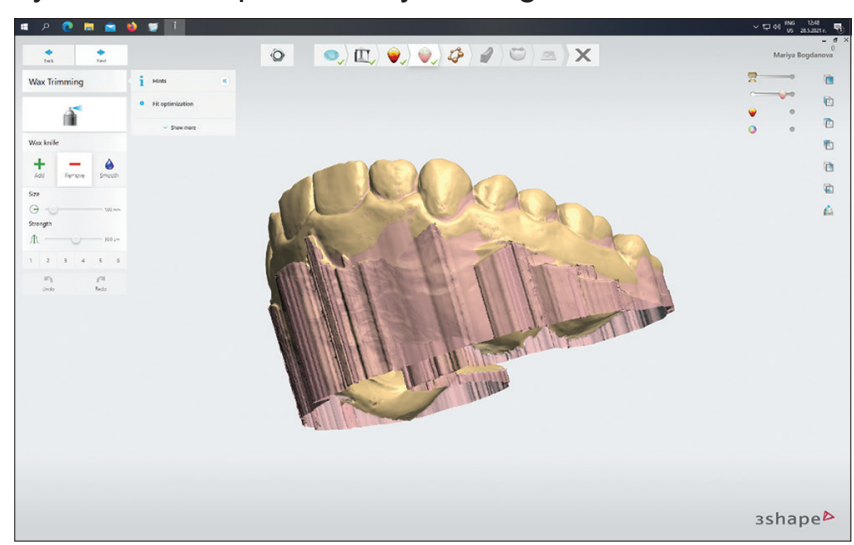

Figure 9: Wax trimming

An important condition is that the first and last points coincide and that the curve is closed. With a slight mouse movement, adjustments are possible before the next step. The screen shows the thickness of the splint, the minimum thickness for the material and the offset of the tooth surfaces, which ensures equal distance between the splint and the contact tooth surfaces, Figures 10 and 11:

\section{surface}

\section{Raise surface - setting the type of occlusal}

There are three software options: raise to antagonist cusp tips (RACT), raise to antagonist plane (RAP), and raise a ramp (RR). The first option (RACT) is not strongly influenced by the occlusal plane set in the first step, because it gives relief to the antagonists in the set position. The other two options (RAP and $\mathrm{RR}$ ) are directly connected to the occlusal plane and create relief next to it. If this plane does not suit us, it is quite possible to adjust it. To set these options requires

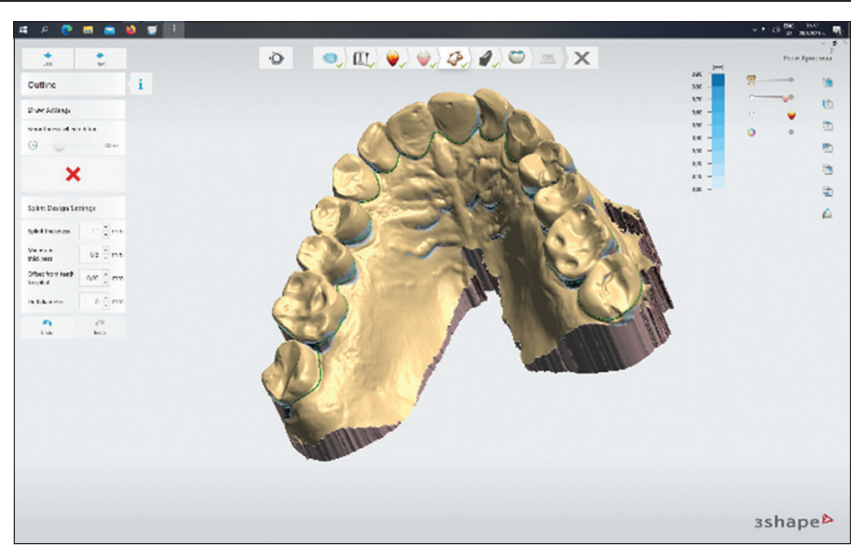

Figure 10: Palatal view of the outline

marking the field in the desired area. If desired and according to clinical indicators, the different options can be combined with each other (eg RACT in the distal areas and RAP in the front), Figures 12 and 13:

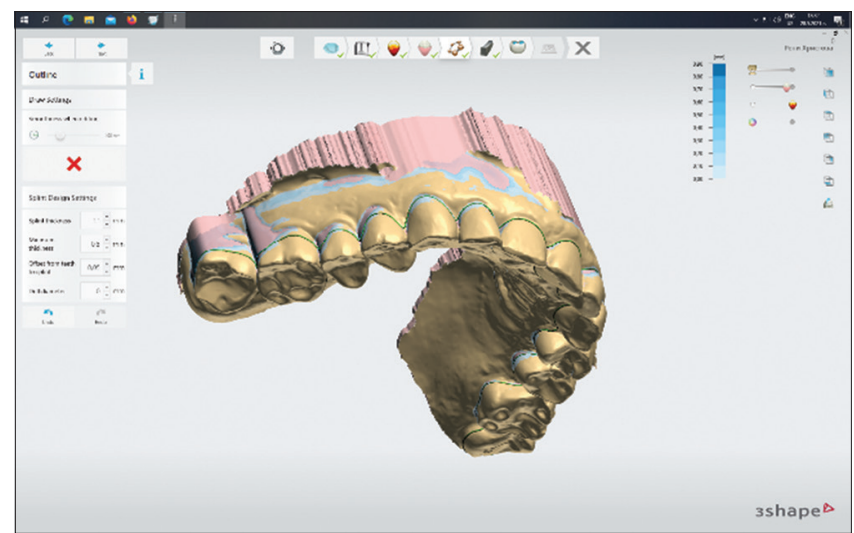

Figure 11: Side view of the outlines

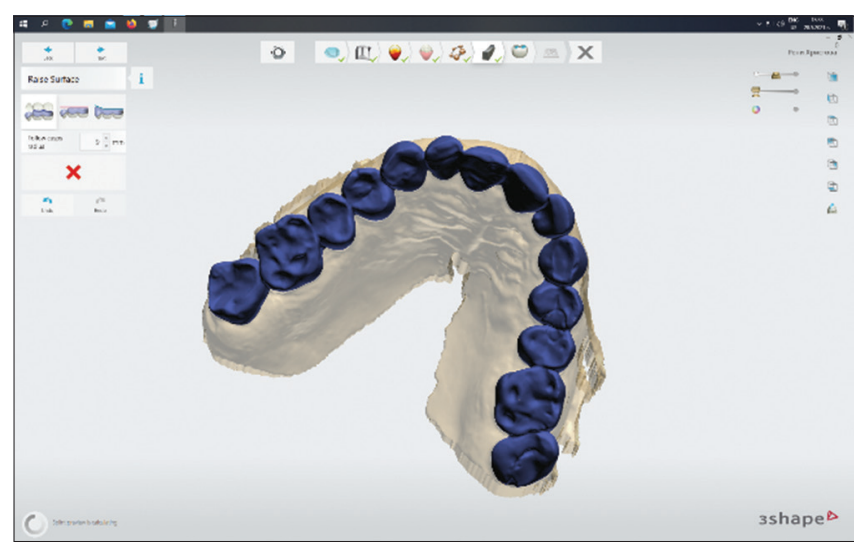

Figure 12: Marked arch with RACT option

\section{Adapt design}

In this step, the already designed splint is displayed. Basically here is the occlusion check. The contacts are visualized with a color-coded scheme. When the virtual articulator is turned on, the lower jaw moves and shows the occluding surfaces in dynamic occlusion. With the available tools, the surface of the 


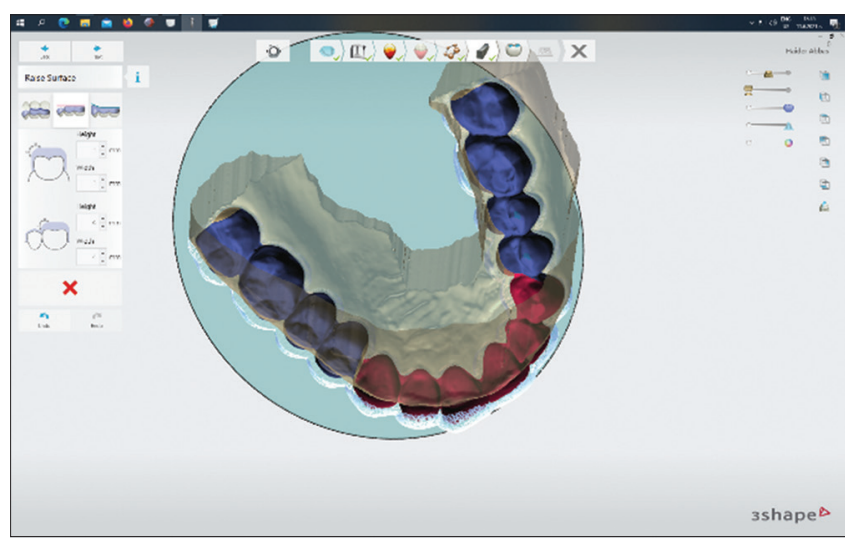

Figure 13: Frontal RAP, distal RACT zones

splint can be added, removed, or smoothed to the desired result. When removing more than the minimum thickness, the software alerts and fills the defect. To check the resulting design and thickness, the model and the splint can be "cut" in the desired direction. In addition to the profile, the occluding accuracy in this section is also demonstrated. When using the respective instrument, measurements can also be made between marked two points, Figures 14 and 15:

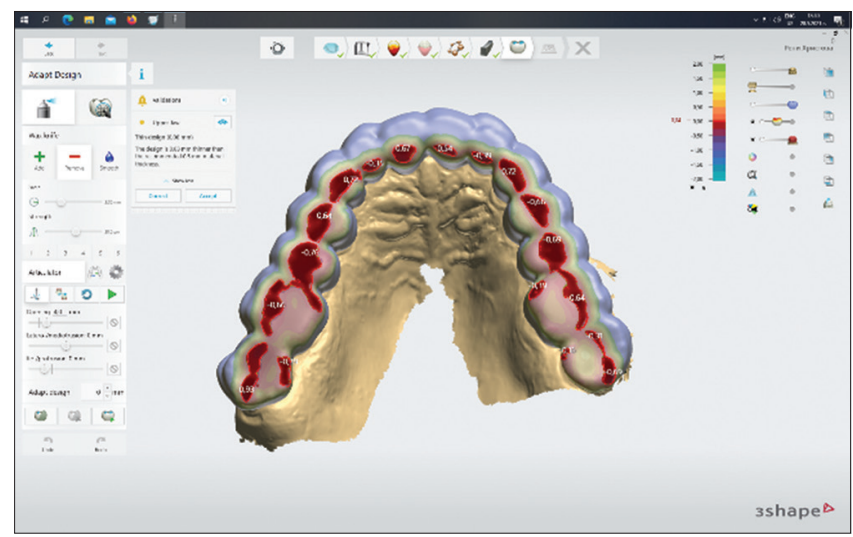

Figure 14: Static occlusion

\section{Production preparation}

This is the final step. In addition to the last view, a positive or negative ID tag with name, number, date, etc., can be added to the splint. Because of the thickness, the software can give only the positive option. If the splint will be made through CAD/CAM milling, for the bur is impossible to cut the small letters. On completion of this step, the created splint is converted into an STL file and sent to the laboratory for printing or cutting, Figures 16.

If, after a clinical adjustment, the splint does not meet expectations, the design can be reset and started again. The scanned upper and lower jaws are preserved, so there is no need to re-scan the patient. Although the design has been canceled, the information for the individual steps has been saved, but confirmation is required. Therefore, the re-design

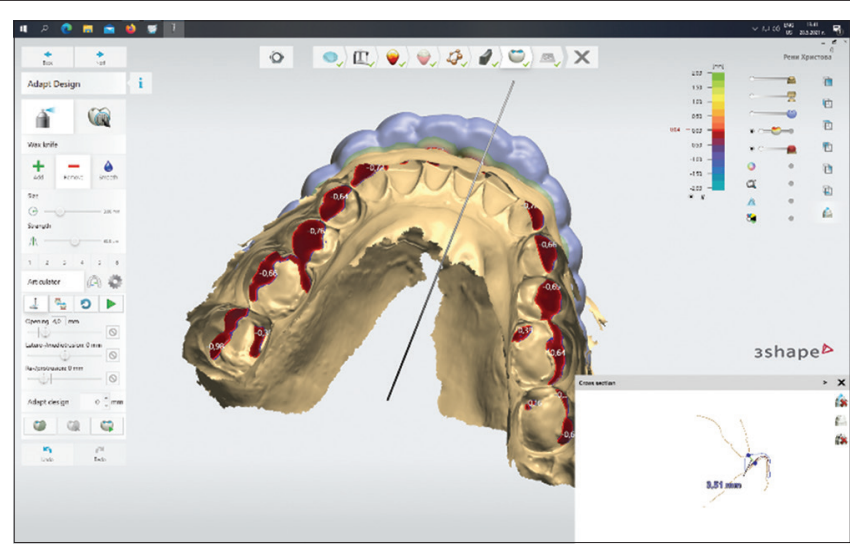

Figure 15: Cut and measurement in frontal area

is obtained significantly faster than the original design.

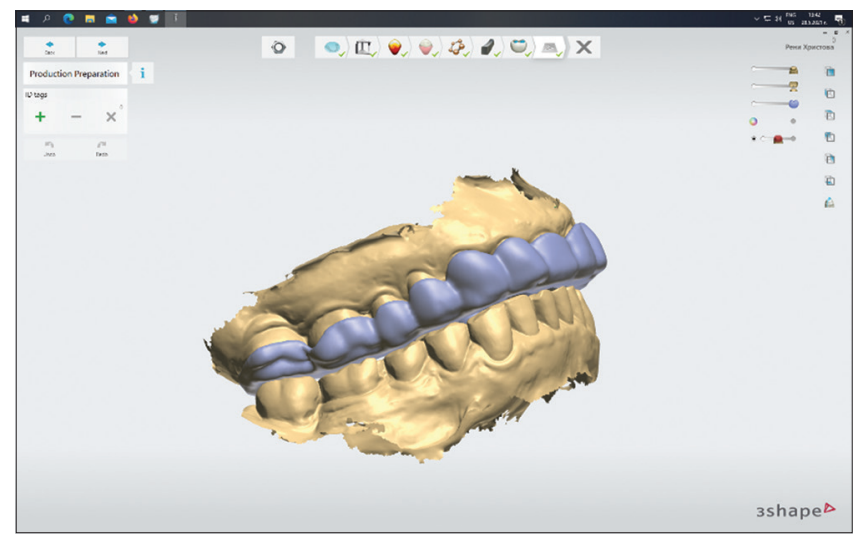

Figure 16: Final occlusal splint

\section{Discussion}

The digital design allows the creation of splints with parameters combining well known types of splints, but for individual clinical situations. Stabilization and repositioning splint are the main classic types. The three software variations (raise to antagonist cusp tips, raise to antagonist plane and raise a ramp), as well as the combination between them completely covers the creation of all splint types [2], [3], [4], [5]. The addition of the virtual articulator and the introduction of additional information, mainly about the condition of TMJ, guarantees the creation of a successful medical product. This is especially necessary in cases of severe clinical symptoms [8], [9]. In addition to the basic parameters created by the software, it is also possible to manually make adjustments by adding, removing or smoothing the splint. Precise and detailed workflow in terms of occlusal relationships and distance from tooth surfaces reduces the clinical time for placement and articulation of the splint [12]. A big advantage of digital technologies is the virtual storage of information. In the event of breakage or loss 
of the splint, the same product can be created within the manufacturing process [13].

\section{Conclusion}

The digital design of splints allows the creation of various modifications according to the specific clinical situation. The eight steps, some of which very short, make it possible to obtain an ultimate virtual object in about $40 \mathrm{~min}$. The design software is not only aimed at dental technicians but also allows dentists to use it.

\section{References}

1. Bumann A, Lotzmann U. TMJ Disorders and Orofacial Pain New York: Thieme Stuttgart; 2002. p. 306-15.

2. Clark GT, Sakai S, Merrill R, Flack VF, McCreary C. Cross-correlation between stress, pain, physical activity, and temporalis muscle EMG in tension-type headache. Cephalalgia. 1995;15(6):511-8, discussion 451. https://doi. org/10.1046/j.1468-2982.1995.1506511.x

PMid:8706116

3. Lotzmann U. In: Freesmeyer WB, editor. Grundbedingungen
Prothetischer Therapie-Die Befunderhebung. Klinische Prothetik. $1^{\text {st }}$ ed. Heidelberg: Hüthig; 1995.

4. Okeson JP. Bell's Orofacial Pains. $5^{\text {th }}$ ed. Chicago: Quintessence; 1995. p. 175.

5. Lotzmann U. Studien zum Einflulß der Okklusalen PräTherapie Auf Die Zentrische Kieferrelation. Berlin: Quintessenz; 1999.

6. Available from: https://www.3shape.com/en/software/splintstudio [Last accessed on 2021 Aug 13].

7. Available from: https://www.exocad.com/our-products/exocaddentalcad/bite-splint-module [Last accessed on 2021 Aug 13].

8. Taneva I, Uzunov T, Apostolov N, Filev N. The digital axiograph-a novel tool in bruxism prevention. In: IOP Conference Series: Materials Science and Engineering. Vol. 1056. Bristol, United Kingdom: IOP Publishing; 2021. p. 012012.

9. Sicat Function Optimization; 2019. Available from: https:// www.sicat.com/products/functional-dentistry/optimotion [Last accessed on 2021 Aug 13].

10. Dawood A, Marti BM., Sauret-Jackson V, Darwood A. 3D printing in dentistry. Br Dent J. 2015;219(11):521-9. https://doi. org/10.1038/sj.bdj.2015.914

PMid:26657435

11. Davidowitz G, Kotick PG. The use of CAD/CAM in dentistry. Dent Clin North Am. 2011;55(3):559-70, 79. https://doi.org/10.1016/j. cden.2011.02.011

PMid:21726690

12. Guzzi EA, Tibbitt MW. Additive manufacturing of precision biomaterials. Adv Mater. 2020;32(13):1901994.

13. Guichet D. Digitally enhanced dentistry: The power of digital design. J Calif Dent Assoc. 2015;43(3):135-41.

PMid:25864301 